\title{
Stocking Decision for Spare Parts in A System with General Demand Distribution
}

\author{
$\mathrm{Xu}$ Tingxue \\ Department of Ordnance Science and Technology \\ Naval Aeronautical and Astronautical University \\ Yantai, China \\ xyx-yt@163.com
}

\author{
Dong Qi* \\ Graduate Students' Brigade \\ Naval Aeronautical and Astronautical University \\ Yantai, China \\ lance0627@163.com \\ * Corresponding author
}

\author{
Zhu Huichuan \\ Graduate Students' Brigade \\ Naval Aeronautical and Astronautical University \\ Yantai, China \\ 721245301@qq.com \\ Zhou Zhiheng \\ Graduate Students' Brigade \\ Naval Aeronautical and Astronautical University \\ Yantai, China \\ zhouzhihengxf@sina.com
}

\begin{abstract}
In order to investigate the stocking decision for a two-echelon inventory system of non-repairable spare parts with multiple demand distributions, we present a universal model to calculate non-repairable spare parts fill rate. Because of the diversity of non-repairable spare parts, the assumption of general demand distribution is made instead of Poisson demand distribution. The two-echelon inventory system consists of a warehouse and several locations. To obtain a high fill rate level of the system in limit cost, an optimization stocking decision model of the system is established by setting total cost as optimization target and systematic fill rate as constraints. The optimal stocking scheme is solved by Genetic Algorithm. Normal distribution and logistic distribution are taken for numerical examples to demonstrate the performance of universal model. The result shows that fill rate of system can be $95.02 \%$ with the minimum total cost. Sensitivity analyses give the relationship between parameters and fill rate. The research result can offer the decision basis for military supply chain.
\end{abstract}

Keywords-inventory; spare parts; fill rate; optimization; demand distribution

\section{INTRODUCTION}

As one of important fields in equipment accommodation indemnification, the research and optimization of spare parts supply chain need be paid more attention with the development of equipments and improvement of operational readiness requirement. Inventory systems usually satisfy demands from more than one type. The types of demands derive the characteristic of spare parts, such as spare parts contain repairable spare parts and non-repairable spare parts. Thus, the real situations drive us to consider the inventory system with multiple demand classes.

The practices of stocking decision face spare parts demands. Most literatures considered an inventory policy for solving the problem of repairable spare parts with Poisson distribution ${ }^{[1-4]}$, since the repairable spare parts usually are valuable to influence the total inventory cost. Wang Naichao et al studied inventory configuration optimization problems in multi-echelon inventory with $(\mathrm{s}-1, \mathrm{~s})$ policy ${ }^{[5]}$; Tao Xiaochuang et al considered fill rate of spare parts as research objective $^{[6]}$; Lee L H and Cheng Hailong analyzed models using expoentially distribution with Poisson demand, and then established a expected cost function ${ }^{[6,7]}$.

Even though there are some models in the literature that incorporate Poisson distribution and $(\mathrm{s}-1, \mathrm{~s})$ policy, there is a lack of studies considering general distributions except for Poisson distribution, as well as other inventory policy except for $(\mathrm{s}-1, \mathrm{~s})$ policy. Three major differences from the literature are outlined here. Firstly, our paper takes the non-repairable spare parts as research objects, which includes diverse types of spare parts and demands. Secondly, our paper establishes a fill rate model with general distributions and $\left(\mathrm{t}_{0}, \mathrm{~s}\right)$ policy. Lastly, we establish a optimization model, and conduct numerical experiments for investigating the optimal stocking decision.

\section{ASSUMPTIONS AND NOTATION}

In this section, a two-echelon system containing $N$ locations $(i=1,2, \mathrm{~L}, N)$ and one center warehouse $(i=0)$ is presented. The system also supplies $n$ types of spare parts. The following notations are used in developing the models.

$C_{j}$ per configuration cost of spare parts $j$ as

$T$ support period of spare parts

$\mathrm{SOH}_{i j}(t)$ inventory level of spare parts $j$ at location $i$ in any fixed interval of length, $t$

$D_{i j}(t)$ demand quantity of spare parts $j$ at location $i$ in any fixed interval of length, $t$

$I N_{i j}(t)$ supply quantity of spare parts $j$ at location $i$ in any fixed interval of length, $t$

$L_{i j}$ purchase lead time of spare parts $j$ at location $i$ 
The supply process of system can be described as follows: when a failure occurs, it can be replaced by a new one from corresponding location, and the failure one will be disposed. If there is no spare part being replaced at the corresponding location, the backorder will be occurred, and the inventory level of location can be returned to the original level after next period. Therefore, the relationship among $S O H_{i j}, I N_{i j}$ and $D_{i j}$ can be expressed as follows:

$$
S O H_{i j}(t+1)=S O H_{i j}(t)+I N_{i j}\left(t-L_{i j}\right)-D_{i j}(t)
$$

In order to simplify the stocking decision model, some assumptions are defined as follows:

- Equipments contain Line Replaceable Units (LRU), which can be defined as $j=1,2, \mathrm{~L}, J$.

- There is no lateral transshipment among locations.

- Let $D_{t}$ denotes demand distributions, where $t=1,2, \mathrm{~L}$, and are independent identically distribution, the mean value is $\mu$.

- $\quad$ Lead time must be considered.

- Supply time and delay time can be neglected.

- Lead time must be considered.

- Equipments in system are identical.

\section{SySTEMATIC FILL RATE MODEL}

\section{A. Fill Rate Definition}

According to $\left(\mathrm{t}_{0}, \mathrm{~s}\right)$ policy, we define $a \mid b$ as $a$ modulo $b$ for integers $a$ and $b$, so $t \mid t_{0}=0$ can be denoted as a review period, which including $0, t_{0}, 2 t_{0}, \mathrm{~L}$. Meanwhile, the definition of fill rate can be described as the fraction of demand that can be satisfied immediately from on-hand inventory, so its model can be established as $(2)^{[8-9]}$

$$
P=\lim _{T \rightarrow \infty} E\left[\frac{\sum_{t=1}^{T} \min \left\{(\operatorname{SOH}(t)+I N(t-L))^{+}, D(t)\right\}}{\sum_{t=1}^{T} D(t)}\right]
$$

where $S_{I N}^{+}(t)=\max \left\{S_{I N}, 0\right\}$.

\section{B. Fill Rate Model based on General Distribution}

For the proposed system in this paper, the fill rate model on spare part $j$ at location $i$ can be (3).

$$
P_{i j}=\lim _{T \rightarrow \infty} E\left[\frac{\sum_{t=1}^{T} \min \left\{S_{I N_{i j}}^{+}(t), D_{i j}(t)\right\}}{\sum_{t=1}^{T} D_{i j}(t)}\right]
$$

For (3), it is too difficult to calculate spare parts fill rate with practical data. Thus, (3) must be transformed as (4), which refers to renewal theory.

$$
P_{i j}==\frac{1}{t_{0} \mu_{i j}} \lim _{T \rightarrow \infty} E\left\{\sum_{k=0}^{t_{0}-1} \frac{\left[\sum_{t \in\{t:(t-L) \mid R=k\}} \min \left\{S_{I N_{i j}}^{+}(t), D_{i j}(t)\right\}\right]}{T / t_{0}}\right\}
$$

where $\left(t-L_{i j}\right) \mid t_{0}=0,1, \mathrm{~L}, t_{0}$ when $t \geq L_{i j}$.
The supply quantity is determined by periodical demand and on-hand level at a location, so

$$
S_{I N_{i j}}(t)=S_{i j}-\sum_{k=1}^{L_{i j}+\left[\left(t-L_{i j}\right) \mid R\right]} D_{i j}(t-k)
$$

According to (4) and (5), $P_{i j}$ can be obtained by (6).

$$
\begin{aligned}
P_{i j} & =\frac{1}{t_{0} \mu_{i j}} E\left[\sum_{k=1}^{t_{0}-1} \min \left\{\left(S_{i j}-\sum_{m=1}^{L_{i j}+k} D_{i j}(m)\right)^{+}, D_{i j}\left(L_{i j}+k+1\right)\right\}\right] \\
& =\frac{1}{t_{0} \mu_{i j}} \sum_{k=0}^{t_{0}-1}\left(\mu_{i j}-E\left\{\left[D_{i j}\left(L_{i j}+k+1\right)-\left(S_{i j}-\sum_{m=1}^{L_{i j}+k} D_{i j}(m)\right)^{+}\right]\right\}\right) \\
& =1-\frac{1}{t_{0} \mu_{i j}} \sum_{k=0}^{t_{0}-1}\left\{E\left\{\left[D_{i j}\left(L_{i j}+k+1\right)-\left(S_{i j}-\sum_{m=1}^{L_{i j}+k} D_{i j}(m)\right)^{+}\right]^{+}\right\}\right\}
\end{aligned}
$$

Let $K(S)=\mu_{i j}\left[1-P_{i j}(S)\right]$, then

$$
\begin{aligned}
K\left(S_{i j}\right) & =\frac{1}{t_{0}} \sum_{k=0}^{t_{0}-1}\left\{\mu_{i j}\left[1-F^{\left(L_{i j}+k\right)}\left(S_{i j}\right)\right]\right. \\
& \left.+\int_{0}^{\infty} \int_{S_{i j}-a}^{S_{i j}}\left(a+b-S_{i j}\right) \mathrm{d} F^{\left(L_{i j}+k\right)}(b) \mathrm{d} F(a)\right\}
\end{aligned}
$$

where $F^{\left(L_{i j}+k\right)}()$ can be the distribution function of $\sum_{j=1}^{L_{i j}+k} D_{j}$.

According to Leibnitz formula ${ }^{[10]}$, then

$$
K^{\prime}\left(S_{i j}\right)=\frac{1}{t_{0}}\left[F^{\left(L_{i j}+k\right)}\left(S_{i j}\right)-F^{L_{i j}}\left(S_{i j}\right)\right]
$$

Since $P_{i j}(0)=0, K(0)=\mu_{i j}\left[1-P_{i j}(0)\right], P_{i j}$ can be

$$
\begin{aligned}
P_{i j}\left(S_{i j}\right) & =1-\left[K(0)+\int_{0}^{S_{i j}} K^{\prime}(a) \mathrm{d} a\right] / \mu_{i j} \\
& =\frac{1}{\mu_{i j} t_{0}} \int_{0}^{S_{i j}}\left[F^{L_{i j}}(b)-F^{\left(L_{i j}+t_{0}\right)}(b)\right] \mathrm{d} b
\end{aligned}
$$

Obviously, fill rate $P_{i j}$ can be determined by parameters, such as inventory level $S_{i j}$, demand distribution $F($ ), review period $t_{0}$ and lead time $L_{i j}$.

The general model of fill rate usually is not applied in practice, because the model needs numerous data to calculate fill rate. However, there is only a small sample of data being collected, demand distribution assumption can be made before model calculation. For non-repairable spare parts, it is irrational to make assumption that the demand distribution is Poisson distribution, since the demand is always in a batch ${ }^{[11-13]}$. So it is assumed that demand distribution of non-repairable spare parts can be Normal distribution, log-Normal distribution or logistic distribution.

Logistic distribution is close to Normal distribution. When the form of Normal distribution is $\mathrm{N}(\mu, \sigma)$, where $\mu$ denotes mean value and $\sigma$ denotes standard deviation. For Logistic distribution, its cumulative distribution function follows ${ }^{[14]}$. 


$$
F(x)=\frac{1}{1+\mathrm{e}^{-(x-m) / r}}
$$

where $m=\mu ; r=\sigma \sqrt{3} / \pi$.

So the fill rate model of logistic distribution can be obtained by (11).

$$
\begin{aligned}
P_{i j}\left(S_{i j}\right) & =\frac{1}{\mu_{i j} t_{0} \pi}\left[\sigma \sqrt{3 L_{i j}} \log \left(1+\mathrm{e}^{\left(S_{i j}-L_{i j} \mu_{i j}\right) \pi / \sigma \sqrt{3 L_{i j}}}\right)\right. \\
& \left.-\sigma \sqrt{3\left(L_{i j}+t_{0}\right)} \log \left(1+\mathrm{e}^{\left(S_{i j}-\left(L_{i j}+t_{0}\right) \mu_{i j}\right) \pi / \sigma \sqrt{3\left(L_{i j}+t_{0}\right)}}\right)\right]
\end{aligned}
$$

As above mentioned, the fill rate model of Normal distribution also can be obtained in similar way.

\section{Stocking Decision Optimization Model}

\section{A. Model Establishment}

Systematic fill rate model can be established as (12) according to [15-16].

$$
P=\sum_{i=1}^{J} \sum_{i=0}^{N} \frac{\mu_{i j}}{\sum_{j=1}^{J} \sum_{i=0}^{N} \mu_{i j}} P_{i j}
$$

where $P$ can be systematic fill rate; $i=0,1,2, \mathrm{~L}, N$; $j=1,2, \mathrm{~L}, J$.

In this section we extend this research to handle the inventory problem, which can be considered to be an optimization problem with a deterministic objective function and multiple stochastic constraints. The stocking decision optimization model can be established as (13) and (14).

$$
\begin{aligned}
& \min C=\min \sum_{i=1}^{N} \sum_{j=1}^{J} S_{i j} C_{i j} \\
& \text { s.t. }\left\{\begin{array}{l}
P \geq P_{0} \\
P_{i j}\left(S_{i j}\right) \geq P_{0 j} \\
t_{0}>L_{i j}>0 \\
L_{i j}+t_{0}=\mathrm{n}_{j}\left(\mathrm{n}_{j}>0\right)
\end{array}\right.
\end{aligned}
$$$$
i=0,1,2, \mathrm{~L}, N ; j=1,2, \mathrm{~L}, J \text {. }
$$

Where $C$ denotes the total cost; $P_{0 j}$ denotes the lower bound of fill rate on $j ; \mathrm{n}_{j}$ can be constant.

\section{B. Optimization Algorithm}

Although marginal algorithm is often used to solve the spare parts optimization problem, it can hardly tackle above model, since the model is too complex to calculate. We thus propose using a genetic algorithm (GA), which is one kind of metaheuristics, and develop a GA-based algorithm when given a large amount of sampling budget.

In what follows the main solution process of the GA employed in this research are described, Fig .1 shows the program flowchart of the proposed $\mathrm{GA}^{[17]}$.

- There are $(N+1) \times J$ parameters in objective function, multi-parameter cascade coding may be applied. According to constraints, the limited research space method is used to determine the range of parameters.
- For optimization model, it can be adopted to establish fitness function: $f=1 / \sum_{i=1}^{N} \sum_{j=1}^{J} S_{i j} C_{i j}$.

- Identifying selection operators. All individuals can be chosen in probability $p_{\mathrm{m}}$ to make crossover. Then, the chosen number can be identified by simulated roulette wheel operation.

- Crossover operators are employed in one-point crossover strategy. For each pair of individuals, the parts of two individuals' chromosomes are interchanged in their intersection to produce new individuals, according to probability $p_{\mathrm{c}}$.

- Mutation. The original gene value may be replaced in the mutation locations of individuals, which are chosen by random sampling with default probability, and new populations and individuals can be produced.

- The last step in a GA is to check whether the algorithm has found a solution that is good enough to meet the expectations. Stopping criterion in this research is default evaluation number.

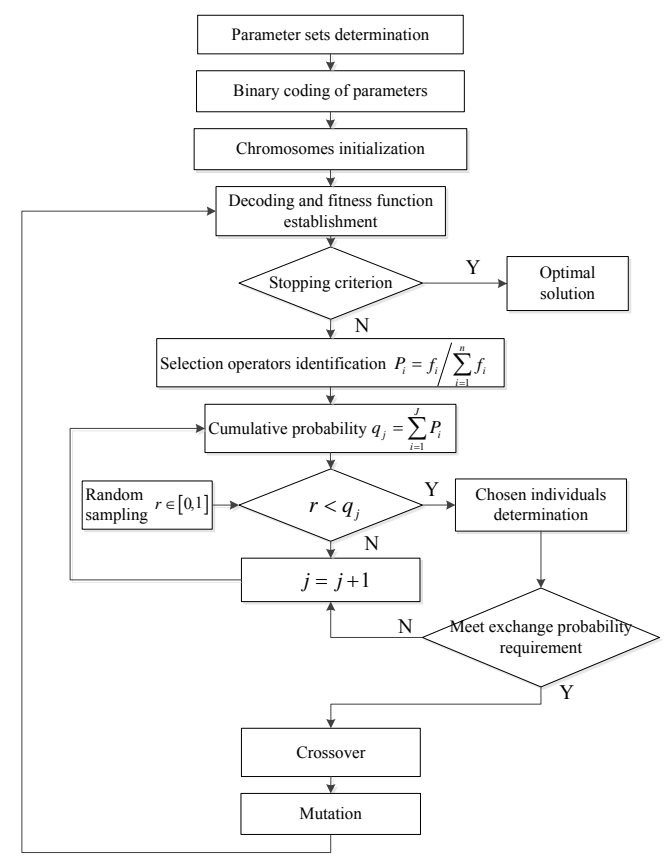

Figure 1. Flowchart of Genetic Algorithm.

\section{NUMERICAL STUDY}

To assess the quality and accuracy of the proposed model and algorithm, a numerical study have been performed. The system under study contains one warehouse $B_{0}$ and three locations $B_{1}, B_{2}, B_{3}$, which $B_{0}$ supplies spare parts to three locations. The spare parts are non-repairable, and are denoted as $\mathrm{LRU}_{1}, \mathrm{LRU}_{2}, \mathrm{LRU}_{3}$. Take Normal distribution and logistic distribution for example, the demand of locations are independent Demand of $\mathrm{LRU}_{1}$ is assumed as logistic distribution and that of $\mathrm{LRU}_{2}, \mathrm{LRU}_{3}$ is assumed as Normal distribution The supply period is assumed to be constant at 180 days. The requirement for systematic fill rate is not below 0.95 . 
The corresponding parameters of example are shown in TABLE I .

TABLE I. EXAMPLE PARAMETERS

\begin{tabular}{|c|c|c|c|c|c|c|c|c|}
\hline \multirow{2}{*}{$\begin{array}{c}\text { Spare Parts } \\
\text { Number }\end{array}$} & \multicolumn{3}{|c|}{ Demand Distribution Parameters } & \multirow{2}{*}{$L$} & \multirow{2}{*}{$t_{0}$} & \multirow{2}{*}{$C$} & $P_{0 j}$ \\
\hline & $\boldsymbol{B}_{\boldsymbol{I}}$ & $\boldsymbol{B}_{\boldsymbol{2}}$ & $\boldsymbol{B}_{3}$ & $\boldsymbol{B}_{\boldsymbol{0}}$ & & & \\
\hline $\mathrm{LRU}_{1}$ & $\log (1300,12)$ & $\mathrm{Log}(1600,9)$ & $\mathrm{Log}(2400,20)$ & $\log (5300,25)$ & 4 & 180 & 50 & 0.90 \\
\hline $\mathrm{LRU}_{2}$ & $\mathrm{~N}(1800,50)$ & $\mathrm{N}(2600,62)$ & $\mathrm{N}(2520,50)$ & $\mathrm{N}(6920,94)$ & 9 & 180 & 150 & 0.95 \\
\hline $\mathrm{LRU}_{3}$ & $\mathrm{~N}(3350,60)$ & $\mathrm{N}(1930,40)$ & $\mathrm{N}(2850,20)$ & $\mathrm{N}(8130,75)$ & 9 & 180 & 200 & 0.95 \\
\hline
\end{tabular}

According to data in TABLE I, the optimization stocking scheme of the system is obtained by proposed GA program, which solve model (13) and (14). Thus, Table 2 shows the optimization stocking scheme, where the systematic fill rate meet the requirement, $P=0.9502$; the total cost equals 5.825 million yuan. At the same time, the fill rates of locations also meet the requirements respectively. Fig .2 shows the relationship between total cost and systematic fill rate.

TABLE II. OPTIMIZATION STOCKING SCHEME

\begin{tabular}{|c|c|c|c|c|}
\hline Location & LRU1 & LRU2 & LRU3 & $\begin{array}{c}\text { Cost } \\
\text { /Million Yuan }\end{array}$ \\
\hline $\mathrm{B}_{1}$ & 1261 & 1801 & 3350 & 1.00320 \\
\hline $\mathrm{B}_{2}$ & 1552 & 2601 & 1931 & 0.85395 \\
\hline $\mathrm{B}_{3}$ & 2328 & 2520 & 2850 & 1.06700 \\
\hline $\mathrm{B}_{0}$ & 5141 & 6920 & 8130 & 2.92105 \\
\hline Total & - & - & - & 5.82520 \\
\hline
\end{tabular}

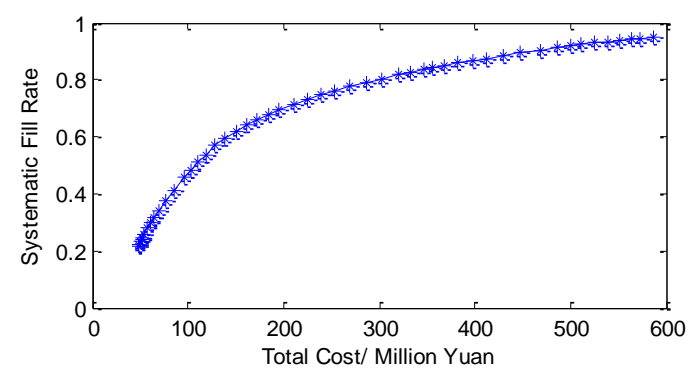

Figure 2. Relationship between systematic fill rate and total cost.

From Fig .2, the systematic fill rate increase along with the total cost, but amplitude decrease gradually, which is the same as that of locations.

In what follows, we present sensitivity analyses for systematic fill rate by simulations. For optimization model, to fixed inventory level, systematic fill rate will increase when $L_{i j}$ decreasing and $t_{0}$ remaining the same, because the less $L_{i j}$, the faster supply will be serviced to system. Furthermore, if $L_{i j}+t_{0}$ equals constant, systematic fill rate increase with less $L_{i j}$.

In order to verify the rationality of proposed model, we use OPUS 10 to calculate the systematic fill rate in the optimal stocking scheme. The result shows the systematic fill rate is above $95 \%$, which is in accordance with that in this paper. Therefore, the proposed model is creditable.

\section{CONCLUSION}

In this paper, we present a two-echelon inventory system. The system consists of a warehouse and locations. We develop a fill rate model for inventory system with general demand, not only Poisson distribution. An optimization inventory decision model is established based on fill rate model. We obtain the optimal inventory level at locations and a warehouse by GA. Numerical example and sensitivity analyses are also provided.

The followings are some possible research issues which may be addressed in the future. Firstly, the current work can be extended by considering a multi-echelon inventory system instead of a two-echelon inventory system. Lastly, only Normal distribution is considered in numerical example. It is interesting to investigate demands consisting of multi-distribution.

\section{ACKNOWLEDGMENT}

The authors would like to thank the two anonymous referees for their helpful comment and suggestions. This research is supported by Natural Science Foundation of Shandong, ZR2011FQ002.

\section{REFERENCES}

[1] Fu Xingfang, Li Jijun, Li Zongzhi, "A stock strategy model for restorable air material based on two-level providing condition," System Engineering- Theory\& Practice, vol. 24, 2004, pp. 111-115.

[2] Zhou Wei, Liu Yajie, Guo Bo, et al, "Initial configuration model about the valuable parts of weapon system based on two-class supply relationship," System Engineering- Theory\& Practice, vol. 31, 2011, pp. 1056-1061.

[3] Zhou Wei, Guo Bo, Zhang Tao, "Initial configuration model for common equipment parts based on two- class supply relationship," System Engineering and Electronics, vol. 33, 2010, pp.89-93.

[4] Ren Min, Chen Quanqing, Shen Zhen, The theory of spare parts supplies [M]. Changsha: National Defense Industry Press, 2013.

[5] Wang Naichao, Kang Rui, "Optimization of multi-echelon repariable item inventory systems with fill rate as objective," Acra Aeronautica ET Astronautica Sinica, vol. 30, 2009, pp. 1043-1047.

[6] Tao Xiaochuang, Guo Linhan, Xiao Boping, et al, "Demand prediction model for spare parts based on fill rate allocation," Acta Armamentar II , vol. 33, 2012, pp. 975-979.

[7] Lee L H, Chew E P, Teng S, et al, "Multi-objective simulationbased evolutionary algorithm for an aircraft spare parts allocation problem," European Journal of Operational Research, vol. 112, 2008, pp. 885-894.

[8] Cheng Hailong, Kang Rui, Long Jun, et al, "Optimization model on support probability of spare part inventory based on margin utility," O. I. Automation, vol. 27, 2008, pp. 37-40.

[9] Ding Dinghao, "The support probability model for repairable spare parts," Electronic Product Reliability and Environmental Testing, vol. 31, 2013, pp. 1-4.

[10] Wong H, Cattrysse D, Oudheusden D V, "Stocking decision for repairable spares pooling in a multi-hub system," International Journal of Production Economics, vol. 93, 2005, pp. 309-317.

[11] Cheng Hailong, Kang Rui, Wei Yi, et al, "Comparation analysis of fill rate and backorder," Journal of Beijing University of Aeronautics ae nd Astronautics, vol. 34, 2008, pp. 580-583.

[12] Christopher M A. Inventory optimization techniques, system vs. item level inventory analysis. Waltham: Raytheon Company IEEE, 2004.

[13] Sherbrooke C C. Optimal inventory modeling of systems: Multiechelon Techniques. Second Edition Boston: Kluwer Academic Publishers, 2004. 
[14] Zhang Jiang, Zhang Jun, "Fill rate of single-stage general periodic review inventory systems," Operations Research Letters, vol. 35, 2007, pp. 503-509.

[15] Sobel M. J, "Fill rate of single-stage and multi-stage supply systems," Manuf. Serv. Oper. Manage, vol. 6, 2004, pp. 41-52.

[16] Zhao Jianzhong, Li Haijun, Ye Wen, et al, "Optimization configuration modeling of spare parts under constraint of improved system spare part fill rate ," Acta Armamentar II , vol. 34, 2013, pp. 1187-1192.

[17] Chang Wenbing, Li Zonghui, "Research on Genetic Algorithm based aircraft spare parts cost optimization," Aircraft Design, vol. 27,2007 , pp. 65-68 